\title{
BMJ Open Cardiac donation after circulatory determination of death: protocol for a mixed-methods study of healthcare provider and public perceptions in Canada
}

\author{
Kimia Honarmand (1) , ${ }^{1}$ lan Ball, ${ }^{1,2}$ Matthew Weiss, ${ }^{3,4}$ Marat Slessarev, ${ }^{1}$ \\ Robert Sibbald, ${ }^{5}$ Aimee Sarti, ${ }^{6}$ Maureen Meade, ${ }^{7}$ Frédérick D'Aragon, ${ }^{8,9}$ \\ Michael Chasse, ${ }^{10}$ John Basmaji, ${ }^{1}$ Jeanna Parsons Leigh ${ }^{11,12}$
}

To cite: Honarmand K, Ball I, Weiss $\mathrm{M}$, et al. Cardiac donation after circulatory determination of death: protocol for a mixedmethods study of healthcare provider and public perceptions in Canada. BMJ Open 2020;10:e033932. doi:10.1136/ bmjopen-2019-033932

- Prepublication history and additional material for this paper are available online. To view these files, please visit the journal online (http://dx.doi. org/10.1136/bmjopen-2019033932).

Received 28 August 2019 Revised 10 May 2020 Accepted 10 June 2020

Check for updates

(C) Author(s) (or their employer(s)) 2020. Re-use permitted under CC BY-NC. No commercial re-use. See rights and permissions. Published by BMJ.

For numbered affiliations see end of article.

Correspondence to

Dr Kimia Honarmand;

kimia.honarmand@medportal.ca

\section{ABSTRACT}

Introduction Cardiac transplantation remains the best treatment for patients with end-stage heart disease that is refractory to medical or device therapies, however, a major challenge for heart transplantation is the persistent discrepancy between the number of patients on waiting lists and the number of available hearts. While other countries (eg, UK, Australia and Belgium) have explored and implemented alternative models of transplantation, such as cardiac donation after circulatory determination of death (DCDD) to alleviate transplantation wait times, ethical concerns have hindered implementation in some countries. This study aims to explore the attitudes and opinions of healthcare providers and the public about cardiac DCDD in order to identify and describe opportunities and challenges in ensuring that proposed cardiac DCDD procedures in Canada are consistent with Canadian values and ethical norms.

Methods and analysis This study will include two parts that will be conducted concurrently. Part 1 is a qualitative study consisting of semi-structured interviews with Canadian healthcare providers who routinely care for organ donors and/or transplant recipients to describe their perceptions about cardiac DCDD. Part 2 is a convergent parallel mixed-methods design consisting of a series of focus groups and follow-up surveys with members of the Canadian general public to describe their perceptions about cardiac DCDD.

Ethics and dissemination This study has been approved by the Research Ethics Board at Western University. The findings will be presented at regional and national conferences and reported in peer-reviewed publications.

\section{INTRODUCTION}

While the majority of organ donors donate after neurological determination of death (NDD; also referred to as 'brain death'), a growing number of organ donors follow the donation after circulatory determination of death (DCDD) pathway. ${ }^{1-3}$ Patients in the DCDD group usually suffer critical illness and
Strengths and limitations of this study

- This study will use qualitative and mixed-methods approaches to describe the perceptions of Canadian healthcare providers and the general public towards cardiac donation after circulatory determination of death (DCDD)

- An in-depth description of the perceptions of Canadian healthcare providers towards cardiac DCDD using a mixed-methods approach and identification of facilitators and barriers along with potential solutions.

- Generation of a rich, in-depth description of the attitudes, opinions and concerns of the Canadian public regarding cardiac DCDD and its implementation in Canada using a mixed-methods approach.

- The findings of this study will inform the development of a framework to facilitate implementation of cardiac DCDD programmes across the country.

- It is not within the scope of this project to provide an in-depth comparison of the opinions and concerns of important specific subgroups within the general Canadian population (eg, indigenous persons, recent immigrants, or specific religious groups).

a decision is made between the healthcare team and substitute decision-makers to withdraw life-sustaining therapy and allow natural death. One key difference between these pathways is that while the heart continues to beat and organs remain perfused from the moment of NDD to organ recovery, the DCDD pathway includes a period of hypoxia, followed by circulatory arrest prior to death determination (after a 5 min 'no-touch' period) and recovery. DCDD accounts for the largest increase in the number of donated organs in Canada and is believed to have the most potential for further increasing the multiorgan donor pool. ${ }^{4}$ 
While it is technically possible to retrieve all organs from DCDD donors that can be retrieved from NDD donors (heart, lungs, kidneys, liver, pancreas), in most countries, cardiac donation has been limited to NDD donors. But the number of patients on cardiac transplant waitlists exceeds the number of available NDD heart donors. ${ }^{4}$ In 2018, 157 patients were on the heart transplant waitlist in Canada $^{1}$ and 3753 patients were on the heart transplant waitlist in the USA. ${ }^{2}$ During the same year, nine patients died before receiving a heart transplant in Canada ${ }^{1}$ and 345 patients died while waiting for a heart transplant in the USA. ${ }^{2}$ Recent successes in cardiac DCDD programmes in the UK, ${ }^{5}$ Australia, ${ }^{6}$ and Belgium ${ }^{7}$ have led to calls for widespread implementation of such a programme in other centres across the world.

\section{Cardiac DCDD protocols}

Current cardiac DCDD programmes have employed two alternative surgical procedures for retrieving hearts from DCDD donors: direct procurement and perfusion (DPP) and normothermic regional perfusion (NRP) ${ }^{8}$ In DPP, after withdrawal of life-sustaining therapy and declaration of death by circulatory criteria, the donor's sternum is opened, and the heart is surgically removed and placed into an ex-situ perfusion device (a heart machine), where a pulsatile pump restores cardiac activity to maintain perfusion during transport. The transplant team then transports the beating heart to the location of the recipient for transplantation. In NRP, after the same process of withdrawal of life-sustaining therapy and declaration of death by circulatory criteria, the donor's sternum is opened, and the central vessels are cannulated. Then, an extracorporeal device is used to restore thoracoabdominal perfusion. This process will allow for circulation of the donor's blood to the thoracic and abdominal organs. To safeguard against restoring perfusion of the brain, vessels that supply the brain are surgically interrupted to prevent circulation of blood to the brain while circulation to the target organs is restored. With NRP, donor cardiac activity is restored prior to surgical removal of the heart, thus permitting in-situ assessment of cardiac function. ${ }^{8}$

Widespread implementation of cardiac DCDD has been slowed partly by questions surrounding the ethics of resuming cardiac activity after declaration of death by circulatory criteria. It has been argued that if the diagnosis of death by circulatory criteria necessitates the irreversible cessation of cardiac activity, its restoration violates the dead donor rule, which states that organ recovery can only occur after death. ${ }^{9-11}$

NRP is possibly more controversial than DPP because it involves restoration of circulation within the donor's body after surgically interrupting the cerebral vasculature to ensure that circulatory flow to the brain is not restored. In some jurisdictions, such as Australia, where the definition of death is based on permanent cessation of systemic circulation, its restoration within the donor body would violate such a definition. ${ }^{12}$ Furthermore, there are concerns about the restoration of cerebral blood flow when thoracoabdominal circulation is restored within the donor body. ${ }^{10}$ There is no consensus on the best methods to ensure that surgical techniques are effective in preventing restoration of any cerebral perfusion nor what amount, if any, such flow would violate the dead donor rule.

Although these ethical considerations have been previously debated within organ donation and transplant communities, these discussions have been largely devoid of any consideration for stakeholder perceptions. ${ }^{13}$ Given any policy change would impact an entire population, and there is no ethical certainty about the practice of cardiac DCDD, it is important to gauge stakeholder perceptions towards cardiac DCDD and its implementation in Canada.

\section{Stakeholder perceptions towards cardiac DCDD}

We conducted a scoping review of the literature exploring the attitudes and opinions of stakeholders (healthcare professionals, donor families, transplant recipients or the general public) on cardiac DCDD and identified no studies in which the attitudes and opinions of stakeholders about cardiac DCDD was described. ${ }^{13}$

To address this critical knowledge gap, we conducted two national web-based surveys to explore stakeholder perceptions about cardiac DCDD. ${ }^{14} 15$ Among 398 healthcare providers, $92 \%$ believe that the DPP approach (in which the heart is retrieved and placed in a perfusion device) to cardiac DCDD is acceptable and 87\% support its implementation in Canada. Similarly, $78 \%$ believe that the NRP approach (in which circulation to thoracoabdominal regions is restarted within the donor's body prior to heart retrieval) to cardiac DCDD is acceptable and $71 \%$ support its implementation in Canada. Participants agreed that there are concerns related to ethical considerations, resource requirements, the quality of the donated heart and the potential for negative impact on other organs being recovered for transplantation. ${ }^{14}$

Among 1001 members of the Canadian public, 84\% believe that the DPP approach to cardiac DCDD is acceptable and $74 \%$ support its implementation in Canada. Similarly, $78 \%$ of Canadians believe that the NRP approach to cardiac DCDD is acceptable and 65\% support its implementation in Canada. ${ }^{15}$

Despite this high level of acceptance, open-ended survey responses revealed several areas of concern among the Canadian public that recurred in our thematic analysis. There were concerns around:

- The certainty of death determination: 'I am concerned that people may not actually be dead.'

- The restoration of thoracoabdominal circulation in NRP: '[ $\mathrm{I} \mathrm{am}]$ uncomfortable with restarting heart within body. If this is possible then why not just treat patient?'

- The possibility of brain reperfusion in NRP: "What if blood made it through and the brain awoke?", and "it would be fine if the brain is not reactivated.'

- The ligation of the central vessels to prevent cerebral perfusion: 'The proposed procedure of tying off 


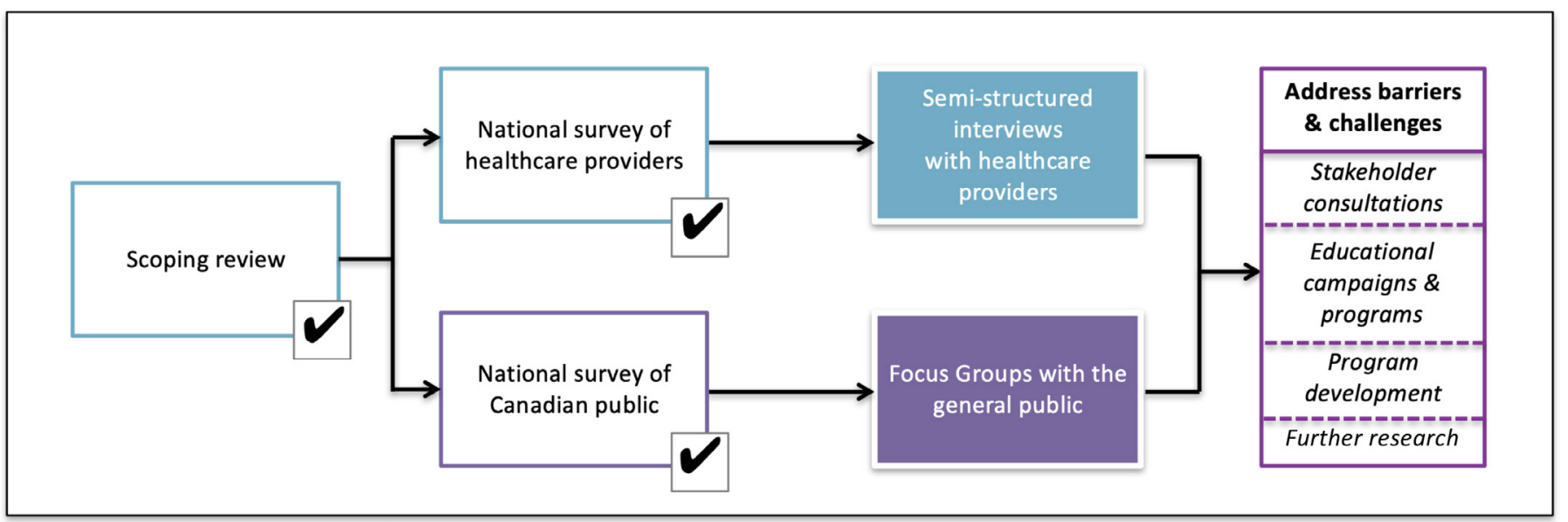

Figure 1 Overview of the overall programme of research describing stakeholder perceptions towards cardiac donation after circulatory determination of death.

the vessels to the brain ...seem[s] quite invasive and family/decision makers may not approve.'

These concerns, if not addressed during the implementation of cardiac DCDD programmes in Canada, could erode public trust in deceased organ donation with potentially negative effects on consent rates and future organ donation research. Similarly, the ethical and practical concerns expressed by the majority of healthcare providers across various groups suggest that the acceptance for cardiac DCDD in Canada is far from settled among healthcare providers in Canada.

The findings of these surveys have been influential in shaping further discourse and research about the acceptability of cardiac DCDD among healthcare providers and the Canadian public. However, survey methodology does not permit the assessment of respondents' comprehension of complex topics and does not allow for in-depth probing or follow-up inquiries to understand respondent thought processes. Qualitative and mixed-methods approaches are far better suited to elucidate a rich, in-depth description of the perceptions of Canadians.

\section{Objectives}

The proposed study is part of a programme of research (depicted in figure 1) that aims to develop a framework for the implementation of cardiac DCDD programmes across Canada that are acceptable to, and consistent with, the values of Canadians. Building on the results of our national survey, we will conduct a study that prioritises engagement and promotes bilateral dialogue between the organ donation and transplantation communities and the general public in Canada. The specific objectives of this study are to:

1. Describe the attitudes, opinions and concerns of Canadian healthcare providers involved in the management of organ donors and/or transplant recipients on cardiac DCDD and to identify facilitators and barriers to widespread implementation of such programmes in Canada.
2. Describe the attitudes, opinions and concerns of the Canadian general public on cardiac DCDD and its implementation in Canada.

\section{METHODS AND ANALYSIS}

\section{Study design}

The purpose of this study is to describe the perspectives of Canadians regarding cardiac DCDD by using a mixedmethods approach which involves the merging of both qualitative and quantitative data ${ }^{16}$ (figure 2 ). In part 1 , we will conduct a qualitative study to describe the perspectives of Canadian healthcare providers towards cardiac DCDD through semi-structured interviews. In part 2, we will conduct a concurrent mixed-methods study ${ }^{16}$ to describe the perceptions of the Canadian general public using focus groups followed by a survey of focus group participants to contextualise the qualitative data collected as part of the focus groups in terms of participants' individual perspectives towards cardiac DCDD protocols.

\section{Patient and public involvement}

Public advisors (members of the Canadian public without medical training) reviewed the protocol for this study and participated in the development and refinement of the educational material that will be used for the focus groups with members of the general public and video clip development.

\section{Part 1: semi-structured interviews with healthcare providers} Sampling and recruitment

We will conduct approximately 50 individual semistructured interviews with the following groups of healthcare providers who routinely care for organ donors and/ or transplant recipients: (1) transplant physicians and surgeons (transplant cardiologists: $\mathrm{n}=5-10$, transplant cardiac surgeons: $n=5-10$; thoracic/abdominal transplant surgeons: $\mathrm{n}=5-10$ ), (2) organ donation physicians (critical care physicians who are specially trained as organ donation experts; $n=5-10$ ), (3) donation coordinators 


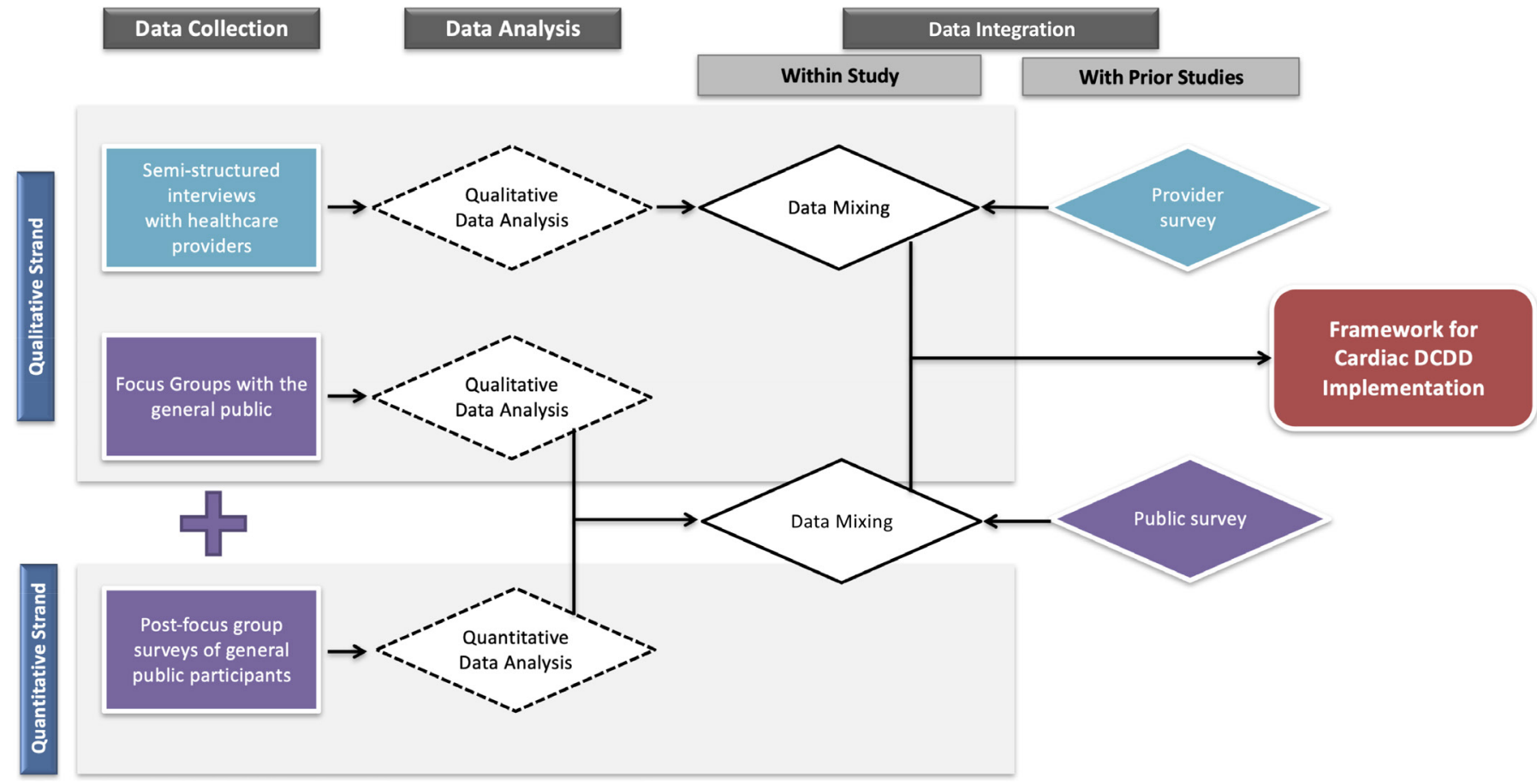

Figure 2 Overview of the procedures and products of the proposed study. DCDD, donation after circulatory determination of death.

(critical care nurses who are specialty trained to discuss organ donation with patients' substitute decision-makers and help to coordinate all activities related to the donation process; $\mathrm{n}=5-7$ ) (4) critical care physicians (who manage the care of deceased or dying potential organ donors in the intensive care unit (ICU) but who are not designated as donation physicians; $n=5-10$ ), (5) ICU nurses $(n=5-7),(6)$ transplant coordinators (nurses who assist in the retrieval of organs in the operating room; $\mathrm{n}=5-7)$, (7) perfusionists $(\mathrm{n}=5-7)$ and (8) cardiac anaesthetists $(\mathrm{n}=5-7)$.

We will purposefully recruit healthcare providers through their respective institutions/departments in a manner that ensures adequate representation of demographic factors including sex/gender and geographical region to maximise the diversity of perspectives captured. Although we plan to conduct approximately 50 interviews across all professional groups, data analysis will be conducted on an ongoing basis and we are prepared to conduct additional interviews until thematic saturation has been achieved. ${ }^{17}$

\section{Interview guide}

We developed an interview guide consisting of prompting, probing and follow-up questions to guide the interview process. These questions are based on themes that emerged from our scoping review ${ }^{13}$ and our national survey of healthcare providers ${ }^{14} 15$ about concerns, expected barriers and facilitators to cardiac DCDD. We also tailored interview themes, questions and language for specific participant groups; for example, adding technical questions about surgery for interviews with transplant surgeons. Prior to launching the interviews, experts in organ donation and transplantation reviewed the interview guide content and clarity, providing feedback for final revisions. The interview guide is provided in online supplementary material A.

\section{Procedures}

Interviews will use web-based video conferencing platforms (eg, Skype, Zoom). Prior to launching the interviews, will conduct five pilot interviews with healthcare providers across Canada to further assess and refine the interview guide. The findings of the pilot interviews will be incorporated into the overall study findings. At the start of each interview, participants will read a concise and pretested summary of cardiac donation in the context of circulatory death. Thereafter, the interviewer $(\mathrm{KH})$ will present specific questions, elicit open-ended responses and ask follow-up questions to further explore all statements. Each interview will last 30 to $45 \mathrm{~min}$.

\section{Data analysis}

All interviews will be audio recorded, transcribed verbatim by a professional transcriptionist, and reviewed for accuracy by one investigator. Two investigators will undertake thematic analysis ${ }^{18}$ by independently conducting lineby-line coding of the transcripts and formulating provisional codes and themes, which will be refined through weekly meetings. This process will generate themes and subthemes that describe participants' attitudes, opinions and concerns related to cardiac DCDD protocols as well as facilitators and barriers to their implementation in Canada. The findings will be contextualised based on the professional characteristics and geographical location of participants. 
Part 2: focus groups with members of the general public Sampling and recruitment

We plan to conduct 12 focus groups, each consisting of 6-8 Canadians residing in four major cities (Montreal, Quebec; Toronto, Ontario; Calgary, Alberta; and Vancouver, British Columbia). We will purposefully sample members of the general public, aiming to achieve a representative sample from each province with respect to gender, age groups, ethnicity, and religious affiliation.

A professional recruitment agency/company will recruit participants through advertisements and by telephoning potential participants from a database of panellists. Participants will be eligible to partake in the study if they are 18 years of age or older, currently living in Canada, fluent in spoken English (or French in Quebec), and able and willing to provide written informed consent to participate in the study. Participants will receive a financial incentive for their participation.

Data analysis will be ongoing and we will be prepared to conduct additional focus groups until thematic saturation has been achieved. ${ }^{17}$

\section{Focus group educational content and discussion guide}

We developed a series of educational content to provide participants with basic information on the following topics: (1) cardiac transplantation, (2) non-cardiac DCDD, (3) cardiac DCDD using the DPP protocol (in which the heart is retrieved and placed in a perfusion device) and (4) cardiac DCDD using the NRP protocol (in which circulation to thoracoabdominal regions is restarted within the donor's body prior to heart retrieval). The development of the educational series was informed by a review of the existing literature on cardiac DCDD protocols and ethical challenges.

The educational content has been rigorously pretested among healthcare professionals with content or process expertise to ensure accuracy and comprehensiveness. It has also been pretested with 18 community advisors (members of the Canadian public with no medical expertise) to ensure that the content is coherent and understandable for the lay public. In addition, we conducted cognitive interviews with three members of the Canadian public to ensure that the content is comprehensible.

The educational content will be prerecorded into a small series of brief video clips, which will be presented to participants during focus groups. Video motion graphics will be used to demonstrate the procedures involved in DPP and NRP using animated illustrations. All investigators will be consulted in the production of the videos at all stages of development to ensure that they accurately reflect the processes and procedures that they are intended to portray. We will also consult with community advisors during the early stages of video development through to video editing to ensure that the videos are comprehensible to the lay public. Closed captioning will be provided. The video clips will be pilot tested prior to the start of the study.
The educational video clips will be presented to participants during the focus groups. This will ensure consistency in content presentation across various focus groups (and avoids portrayal of any biases by the facilitator) and may enhance participant engagement. In addition, such animations were preferred over 'real-life' portrayals of the procedures by members of the general public whom we consulted as part of our national survey studies, with the latter deemed to be potentially uncomfortable to view for some members of the general public. Related questions (prompts, probing and follow-up) will be used to further explore the rationale for participants' perspectives, generating a richer depth of discussion among participants regarding the facilitators and barriers of cardiac DCDD acceptability. These questions have been adapted from our national survey and expanded to delve more deeply into the attitudes, perceptions and concerns expressed by respondents, particularly in free-text comment sections of the survey. The focus group discussion guide is presented is online supplementary material $\mathrm{B}$.

\section{Study setting}

Focus groups will take place in person at specialised facilities in four Canadian provinces (British Columbia, Alberta, Ontario and Quebec). These provinces were targeted for study recruitment due to their high volumes of cardiac transplants and readiness for implementation of cardiac DCDD programmes.

\section{Procedures}

One investigator with training in focus group methodology $(\mathrm{KH})$ and a research associate will facilitate the focus groups in consultation from experts in qualitative research methodologies (JPL and AS). Focus group methodology is ideally suited to achieve our objectives because it provides a deeper level of insight and understanding of a phenomenon by encouraging debate and discussion on a topic not previously familiar to participants. ${ }^{19}$

Prior to launching the focus groups, we will conduct two pilot sessions each with 3-5 Canadians residing in Ontario. This step will allow us to further assess and refine the educational video clips and the focus group discussion guide to ensure that both are clear, concise and appropriate for use in a focus group format.

To allow for a description of the study sample, participants will first be asked to complete a brief survey consisting of 12 Likert-like items where we will collect the demographic characteristics of participants and their previous experiences and self-rated knowledge about organ donation (online supplementary material C).

Focus groups will be facilitated using the focus group discussion guide (online supplementary material B). During the focus group, the facilitator will present each of the educational video clips during and encourage participants to share their perceptions and feelings towards each donation protocol in an unstructured manner whenever possible. The facilitator will also ask a series of open-ended prompting, probing and follow-up questions 
as appropriate to help stimulate a rich discussion and debate among participants (online supplementary material $\mathrm{C}$ ).

At the conclusion of each focus group, participants will be asked to complete a brief survey consisting of 11 Likert-type items that covered the same themes as the focus group discussion guide. Items will explore overall acceptance, willingness to consent for self and willingness to consent on behalf of a family member to non-cardiac DCDD, cardiac DCDD using the DPP protocol, and cardiac DCDD using the NRP protocol. Each item will be followed by an opportunity to provide free-text comments to explain responses (online supplementary material D).

\section{Data analysis}

All focus group discussions will be audio recorded, transcribed verbatim by a professional transcriptionist and reviewed for accuracy by one investigator. Two investigators will undertake thematic analysis ${ }^{18}$ according to the procedures described previously. This process will generate the themes and subthemes that describe the public's attitude towards DCDD in general as well as cardiac DCDD protocols.

For the quantitative (survey) data, we will use descriptive statistics to summarise the demographic characteristics of participants and their perceptions towards DCDD in general as well as cardiac DCDD protocols. We will not conduct inferential statistics given that responses to surveys are intended to elucidate the perceptions of participants after the discussion in which they participated as well as any comments they wish to share confidentially and are not intended to draw any conclusions regarding the attitudes of Canadians in general, a topic that has already been explored in our large-scale national survey study. The findings of the thematic analysis of qualitative data will be contextualised based on participants' demographic characteristics and their survey responses.

\section{Data integration}

We will report the findings of the proposed study independently as well as conduct data integration to include findings from various phases of this programme of research. The findings from the interviews with healthcare providers will be integrated with those of the published healthcare provider survey ${ }^{14}$ to describe healthcare provider perceptions and identify the facilitators and barriers (along with potential solutions) to the implementation of cardiac DCDD programmes at centres across the country, contextualised based on participants' professional roles and geographical region within Canada. The findings from the focus groups with members of the Canadian public will be integrated with those of the published Canadian public survey ${ }^{15}$ to describe public perceptions on various aspects of cardiac DCDD, contextualised based on participants' demographic characteristics and attitudes towards cardiac DCDD protocols as identified by the follow-up surveys. Data from all four studies of this programme of research, the two proposed studies and the two published national surveys, ${ }^{14}{ }^{15}$ will be synthesised to provide a framework that will inform the development of a comprehensive description of stakeholder perceptions, perceived facilitators, and perceived barriers along with any potential solutions that arise from this work.

\section{Validity and methodological rigour}

Several strategies will be used across all stages of this study to enhance the validity, methodological rigour and trustworthiness of the findings of this study according to the approach described by Krefting. ${ }^{20}$ To optimise the credibility of findings, the researchers who will conduct interviews and focus groups and participate in data analysis will engage in reflexive journaling during the study to document and assess their own perspectives that may influence the research process. To further enhance, triangulation will be achieved using two data sources (healthcare providers and the general public) and various data collection methods (interviews with healthcare providers, focus groups with the general public and follow-up surveys of the focus group participants). Furthermore, the researchers will discuss emerging insights and perspectives with coinvestigators and other colleagues as a form of peer debriefing aimed at enhancing credibility.

To enhance transferability, we will provide a thick description of participants and by use of purposive sampling. Dependability will be enhanced by stepwise replication during data analysis by enlisting two members of the research team to participate in coding of a sample of transcripts to identify initial codes that emerge from the data and to develop consensus around the code definitions. Finally, confirmability will be enhanced by the maintenance of a comprehensive audit trail to memorialise all study processes from study design to data analysis to integration, as well as reflexivity and triangulation as previously described. All qualitative findings will be reported in accordance with the Consolidated Criteria for Reporting Qualitative Research checklist. ${ }^{21}$

\section{ETHICS AND DISSEMINATION}

We have obtained approval from the Research Ethics Board (REB) at Western University (WesternREM) for both components of this study (ID numbers 113807 and 113808). All participants will be asked to provide written informed consent to participate in the study. All participants will be asked to provide written informed consent prior to participating in the study.

The findings of both studies will be provided to Canadian Blood Services (the national organisation that oversees organ donation activity in Canada), presented at regional and national meetings and conferences, and prepared as at least two manuscripts for publication in peer-reviewed journals.

\section{Relevance of findings}

There is a discrepancy between the number of available donor hearts and the number of patients on heart 
transplant waitlists. Widespread implementation of cardiac DCDD in Canada has the potential to improve outcomes for patients on the heart transplant waitlist and reduce the heart transplant waitlist by increasing the number of available donor hearts. Implementation of any cardiac DCDD programme requires rigorous planning and examination of its acceptability including stakeholder perceptions, and a comprehensive approach to identifying facilitators that can be capitalised and barriers that may be faced along with exploration of possible solutions to the latter. The proposed studies are part of a programme of research that is the most comprehensive approach in engaging with healthcare providers and the general public regarding any protocol in organ donation and transplantation.

Engagement of the surgical and medical transplant community is of particular interest given their insights into the acceptability of DCDD heart retrieval from the perspective of those who care for recipients, their perceptions of the impact of such practice on non-heart organs, and possible technical and pragmatic facilitators and barriers that may be faced if DCDD donors were to also become heart donors. Engagement of members of the general public is paramount to identifying any specific areas of concern regarding cardiac DCDD protocols, devising appropriate ways to address any misconceptions and knowledge gaps among the public, and ensuring that steps towards this process are consistent with Canadian values. Direct, multifaceted dialogue in a focus group setting is the ideal setting for exploring the Canadian public's perceptions of the complex issues surrounding the ethics of cardiac DCDD programmes. We will develop a comprehensive description of the perceptions of the Canadian public on cardiac DCDD, the important contextual factors that influence those perceptions, and the opportunities and challenges its implementation may bring from the perspectives of the public. This will provide a practical and well-informed framework that integrates the opportunities as well as expected challenges, which will in turn guide the design and development of cardiac DCDD programmes in Canada.

Together, these findings will provide researchers, providers and decision-makers at national and provincial levels with vital information to launch well-informed cardiac DCDD programmes that are consistent with Canadian values based on comprehensive public and provider consultation. Moreover, while the focus of this research is on cardiac DCDD, our model of public and provider engagement may be applied to current and future practice changes and new innovations in organ donation and transplantation. Equipped with the findings of this work, the organ donation and transplant communities will be able to ensure that our cardiac DCDD programmes are conducted in a manner that is acceptable to Canadians and maintains trust in the organ donation system.

\section{Author affiliations}

${ }^{1}$ Department of Medicine, Western University, London, Ontario, Canada
${ }^{2}$ Department of Epidemiology and Biostatistics, Western University, London, Ontario, Canada

${ }^{3} \mathrm{CHU}$ de Québec - Université Laval Research Center, Population Health and Optimal Health Practices Research Unit, Trauma-Emergency-Critical Care Medicine, Université Laval Faculté de Médecine, Québec, Québec, Canada

${ }^{4}$ Transplant Québec, Montreal, Québec, Canada

${ }^{5}$ Department of Family Medicine, Western University, London, Ontario, Canada ${ }^{6}$ Department of Critical Care, The Ottawa Hospital, Ottawa, Ontario, Canada ${ }^{7}$ Department of Medicine, Department of Health Research Methods, Evidence and Impact, McMaster University, Hamilton, Ontario, Canada

${ }^{8}$ Department of Anesthesiology, Universite de Sherbrooke Faculte de Medecine et des Sciences de la Sante, Sherbrooke, Québec, Canada

${ }^{9}$ Centre de Recherche du CHUS, Sherbrooke, Québec, Canada

${ }^{10}$ University of Montreal Research Center, Innovation Hub and Department of

Medicine (Critical Care), University of Montreal, Montreal, Québec, Canada

${ }^{11}$ School of Health Administration, Faculty of Health, Dalhousie University, Halifax,

New Brunswick, Canada

${ }^{12}$ Department of Epidemiology and Biostatistics, Cummings School of Medicine, University of Calgary, Calgary, Alberta, Canada

\section{Twitter lan Ball @Ball}

Acknowledgements The authors would like to thank Rachelle Miele for her assistance with the revisions to this manuscript.

Contributors KH, IB and JPL contributed to the conception and design of this study with input from all other authors. All authors contributed to the refinement of the study design and the preparation of this manuscript.

Funding This work was supported by the Canadian Blood Services Kenneth J Fyke Award.

Competing interests None declared.

Patient consent for publication Not required.

Ethics approval Both components of this study have been approved by the Research Ethics Board at Western University.

Provenance and peer review Not commissioned; externally peer reviewed.

Open access This is an open access article distributed in accordance with the Creative Commons Attribution Non Commercial (CC BY-NC 4.0) license, which permits others to distribute, remix, adapt, build upon this work non-commercially, and license their derivative works on different terms, provided the original work is properly cited, appropriate credit is given, any changes made indicated, and the use is non-commercial. See: http://creativecommons.org/licenses/by-nc/4.0/.

\section{ORCID iD}

Kimia Honarmand http://orcid.org/0000-0002-7583-1445

\section{REFERENCES}

1 Canadian Institutes of Health Information. e-statistics report on transplant, waiting list and donor statistics. Ottawa: ON, 2019. Available: https://www.cihi.ca/sites/default/files/document/2013_ estatsinnewtemplate_en-web.pdf [Accessed Feb 2020].

2 Annual data report 2016 of the U.S. organ procurement and transplantation network and the scientific registry of transplant recipients: transplant data 1994-2003. United States department of health and human services. healthcare systems bureau, division of transplantation, Rockville, MD. Am J Trans 2018;18.

3 National Health Services. Annual report on cardiothoracic organ transplantation: report for 2016/2017. NHS Blood and Transplant, 2018: 150. https://nhsbtdbe.blob.core.windows.net/umbraco-assetscorp/12252/nhsbt-cardiothoracic-transplantation-annual-report2017-2018.pdf

4 Canadian Blood Services. Organ donation and transplantation in Canada: system progress report 2006-2015. Ottawa, ON, 2016: 94. https://blood.ca/sites/default/files/ODT_Report.pdf

5 Messer S, Page A, Axell R, et al. Outcome after heart transplantation from donation after circulatory-determined death donors. $J$ Heart Lung Transplant 2017;36:1311-8.

6 Kumar R, Shekar K, Widdicombe N, et al. Donation after cardiac death in Queensland: review of the pilot project. Anaesth Intensive Care 2012;40:517-22.

7 Noterdaeme T, Detry O, Hans M-F, et al. What is the potential increase in the heart graft pool by cardiac donation after circulatory death? Transpl Int 2013;26:61-6. 
8 White CW, Messer SJ, Large SR, et al. Transplantation of hearts donated after circulatory death. Front Cardiovasc Med 2018;5:1-20.

9 Veatch RM. Donating hearts after cardiac death--reversing the irreversible. N Engl J Med 2008;359:672-3.

10 Eynon CA, Murphy PG, Smith M, et al. Heart transplantation after Declaration of death by cardiorespiratory criteria. $J$ Heart Lung Transplant 2010;29:232-3.

11 Tibballs J, Bhatia N. Transplantation of the heart after circulatory death of the donor: time for a change in law? Med J Aust 2015;203:268-70.

12 Gardiner D, McGee A. Death, permanence and current practice in donation after circulatory death. QJM 2017;110:199-201.

13 Ball IM, Honarmand K, Parsons-Leigh J, et al. Heart recovery after circulatory determination of death: time for public engagement. Can J Anaesth 2019;66:1147-50.

14 Honarmand K, Parsons Leigh J, Basmaji J, et al. Attitudes of healthcare providers towards cardiac donation after circulatory determination of death: a Canadian nation-wide survey. Can $J$ Anaesth 2020;67:301-12.
15 Honarmand K, Parsons Leigh J, Martin CM, et al. Acceptability of cardiac donation after circulatory determination of death: a survey of the Canadian public. Can J Anaesth 2020;67:292-300.

16 Creswell JW. Research design: quantitative and qualitative approaches. 2nd edn. Thousand Oaks, CA: Sage Publications, 2003.

17 Strauss AL. Qualitative analysis for social scientists. Cambridge: Cambridge University Press, 2003.

18 Braun V, Clarke V. What can "thematic analysis" offer health and wellbeing researchers? Int J Qual Stud Health Well-being 2014;9:26152.

19 Morgan M, Kenten C, Deedat S, et al. Increasing the acceptability and rates of organ donation among minority ethnic groups: a programme of observational and evaluative research on donation, transplantation and ethnicity (donate). Programme Grants Appl Res 2016;4:1-196.

20 Krefting L. Rigor in qualitative research: the assessment of trustworthiness. Am J Occup Ther 1991;45:214-22.

21 Tong A, Sainsbury P, Craig J. Consolidated criteria for reporting qualitative research (COREQ): a 32-item checklist for interviews and focus groups. Int J Qual Health Care 2007;19:349-57. 